\title{
Bottom-Up Nanofabrication of Supported Noble Metal Alloy Nanoparticle Arrays for Plasmonics
}

Nugroho, Ferry A. A.; landolo, Beniamino; Wagner, Jakob Birkedal; Langhammer, Christoph

Published in:

A C S Nano

Link to article, DOI:

10.1021/acsnano.5b08057

Publication date:

2016

Document Version

Peer reviewed version

Link back to DTU Orbit

Citation (APA):

Nugroho, F. A. A., landolo, B., Wagner, J. B., \& Langhammer, C. (2016). Bottom-Up Nanofabrication of Supported Noble Metal Alloy Nanoparticle Arrays for Plasmonics. A C S Nano, 10(2), 2871-2879. https://doi.org/10.1021/acsnano.5b08057

\section{General rights}

Copyright and moral rights for the publications made accessible in the public portal are retained by the authors and/or other copyright owners and it is a condition of accessing publications that users recognise and abide by the legal requirements associated with these rights.

- Users may download and print one copy of any publication from the public portal for the purpose of private study or research.

- You may not further distribute the material or use it for any profit-making activity or commercial gain

- You may freely distribute the URL identifying the publication in the public portal 


\section{Bottom-Up Nanofabrication of Supported Noble}

\section{Metal Alloy Nanoparticle Arrays for Plasmonics}

Ferry A. A. Nugroho*,‡,1, Beniamino Iandolo ${ }^{\ddagger, 2}$, Jakob B. Wagner ${ }^{2}$ and Christoph

$$
\text { Langhammer*,1 }
$$

${ }^{1}$ Department of Applied Physics, Chalmers University of Technology, 41296 Göteborg, Sweden

${ }^{2}$ Center for Electron Nanoscopy, Technical University of Denmark, 2800, Kongens Lyngby, Denmark 


\section{ABSTRACT}

Mixing different elements at the nanoscale to obtain alloy nanostructures with fine-tuned physical and chemical properties offers appealing opportunities for nanotechnology and nanoscience. However, despite wide-spread successful application of alloy nanoparticles made by colloidal synthesis in, for example, heterogeneous catalysis, nanoalloy systems are so far only very rarely used in solid state devices and nanoplasmonics related applications. One reason is that such applications require integration in arrays on a surface with compelling demands on nanoparticle arrangement, uniformity in surface coverage, and optimization of the surface density. These cannot be fulfilled even using state-of-the-art self-assembly strategies of colloids. As a solution, we present here a generic bottom-up nanolithography-compatible fabrication approach for large area arrays of alloy nanoparticles on surfaces. To illustrate the concept, we focus on Au-based binary and ternary alloy systems with $\mathrm{Ag}, \mathrm{Cu}$ and $\mathrm{Pd}$, due to their high relevance for nanoplasmonics and complete miscibility, and characterize their optical properties. Moreover, as an example for the relevance of the obtained materials for integration in devices, we demonstrate the superior and hysteresis-free plasmonic hydrogen sensing performance of the AuPd alloy nanoparticle system.

\section{KEYWORDS}

nanofabrication, nanoalloy, alloy nanoparticles, nanoplasmonics, hydrogen sensing 
Synergistic effects of the constituents in metal alloy nanoparticles may significantly broaden their characteristics and enhance their functionality compared to pure analogues. Hence, their rich and interesting physical and chemical properties have for a long time attracted wide interest in, for example, the field of heterogeneous catalysis. ${ }^{1-4}$ Also in the field of nanoplasmonics noble metal alloy nanoparticles have been considered, and the optical properties of colloidal binary alloy nanoparticles, such as AuAg or AuCu, have been studied. ${ }^{5,6}$ Apart from fundamental interest, research in the field is spurred by the significant potential to use alloying as additional handle to control and tailor plasmonic properties in combination with other relevant functionalities such as catalytic activity, magnetic properties or - as in focus here - hydrogen sorption properties. However, despite these significant prospects, alloy nanoparticles and nanostructures are so far seldom used in solid-state devices and nanoplasmonics related applications. We believe that one main contributing reason is that, as the state-of-the-art, alloy nanoparticles are almost exclusively produced via colloidal synthesis, ${ }^{2,5,7,8}$ which has a number of key implications and consequences, in particular with respect to their applicability in devices.

For example, synthesis of noble metal alloy nanoparticles with controlled size larger than $30 \mathrm{~nm}$ has not been successful ${ }^{9-17}$ until the very recent work by Rioux and Meunier, who developed a synthesis route combining a co-reduction and seeded growth approach that yielded spherical AuAg alloy nanoparticles in the $30-150 \mathrm{~nm}$ size range. ${ }^{18}$ However, in nanoplasmonics it is typically nanostructures with a characteristic dimension larger than $50 \mathrm{~nm}$ that are most relevant due to their, for instance, higher scattering efficiencies and larger optical cross sections. ${ }^{19,20}$ Moreover, it is crucial to fabricate nanoparticles with shapes other than spheres in order to tune 
the spectral position of the resonance and craft more complex structures like optical metamaterials and metadevices. ${ }^{21}$

The second important factor is that wet-chemically synthesized alloy nanoparticles naturally come in a solution. However, solid state and typical plasmonics applications often require their integration in arrays on a surface with compelling demands on nanoparticle orientation, uniformity in coverage at the $\mathrm{mm}^{2}$ to $\mathrm{cm}^{2}$ scale, and optimization of the surface density to control both near and far field interactions. ${ }^{22,23}$ Fulfilling these criteria with colloidal nanoparticles, even using state-of-the-art self-assembly strategies, is extremely difficult. Therefore, nanolithography based fabrication methods like electron beam lithography (EBL) or colloidal lithography in different variants, are today widely used workhorses for the integration of nanostructures in solid state devices in general, and in nanoplasmonics in particular, ${ }^{24-26}$ since they offer superior control of the aforementioned critical parameters. However, to date with few exceptions, ${ }^{27,28}$ there are no reports of nanofabricated arrays of alloy nanoparticles with well-defined nanoparticle size, shape and composition that can be made with a reasonable yield in terms of uniformity and covered surface area.

In response to the above, we present a generic bottom-up nanolithography strategy for the fabrication of large-area arrays of plasmonic alloy nanoparticles with excellent control of nanoparticle size, shape and composition. Our approach is based on finely tuned layer-by-layer physical vapor deposition of alloy constituents through a nanofabricated mask, and subsequent annealing. We demonstrate the nanofabrication of large area arrays of binary and ternary Aubased alloy nanoparticles with $\mathrm{Ag}, \mathrm{Cu}$ and $\mathrm{Pd}$. We choose these metals owing to their high 
relevance for plasmonic applications, and because they do not exhibit miscibility gaps in their equilibrium phase diagrams at room temperature. ${ }^{29}$ As a demonstration of the superior functionality that can be achieved by alloying, we further investigate the optical plasmonic hydrogen sensing properties of AuPd binary alloy nanoparticles.

\section{RESULTS}

Our general approach to producing alloy nanoparticles is sketched in Scheme 1. First, we fabricate layered nanostructures of the alloy elements by subsequent evaporation into a supported, pre-fabricated mask. We use our well-established nanofabrication method Hole-Mask Colloidal Lithography (HCL) ${ }^{26}$ to generate the mask (see Methods section for details). Nevertheless, we highlight that our approach is compatible with basically any other nanolithographic mask fabrication technique, e.g. electron beam lithography, photolithography or other colloidal lithography variants such as nanosphere lithography. ${ }^{25}$ The most critical step, to be evaluated and fine-tuned for any chosen nanolithography, is to guarantee a precise alloy composition. This is achieved by careful derivation of the thickness of the deposited layers of each constituent, which is done via simple geometrical considerations (see Supporting Information SI), and by relying on the Ångström resolution of state-of-the-art physical vapor deposition systems in terms of thickness of the grown layers. A crucial factor to consider when estimating the required thickness of the alloy element layers is the fact that the evaporated material continuously builds up on top of the mask and, critically, at the rim of the holes in the mask, eventually causing it to shrink..$^{26,30,31}$ This effect generates a tapered structure of the 
nanoparticle grown on the substrate through the hole in the mask, which has to be taken into account in the layer thickness calculation (see SI).

The next fabrication step is thermal annealing to promote the alloy formation from the layered structure. This process relies on the thermodynamically driven atomic mixing of alloy components that can occur far below their melting temperatures. Therefore, we anticipate formation of homogeneous alloys provided that this is thermodynamically favorable, and that we allow sufficient time at an appropriate temperature to reach the equilibrium state. At the same time, as shown below, the annealing process may also induce a minor change of the aspect ratio of the nanoparticle, while the general shape (here a disk) is retained quite nicely (see also Figure S4 in the SI). This slight aspect ratio change is driven by the particles' appetence to attain its equilibrium shape according to the Wulff rule, which in turn is depending on a number of factors such as wetting of the alloy elements with the substrate, annealing time and temperature. Nonetheless, as shown in Figure S5 in the SI, even anisotropic particles like nanoellipses retain their anisotropy after the annealing. The gas environment during the alloying step is also important and has to be controlled accordingly, with details depending on the targeted alloy. If the alloy is to contain constituents prone to oxidation, annealing in inert or even reducing atmospheres is required to retain the metallic state of the nanoparticles. Annealing will also affect the crystallinity of the particle with a general tendency of recrystallization to occur, which leads to grain growth. To this end, it has been reported that annealing also significantly reduces the line-width of plasmonic resonances. ${ }^{32,33}$ 
To demonstrate and evaluate our new approach we fabricated and characterized series of largearea quasi-random arrays of Au-based binary alloy nanodisks (i.e. AuAg, AuPd and AuCu) with varying compositions ( $(0-100$ at. $\%$ of $\mathrm{Au}$, in steps of 10 at. \%) and pre-annealing dimensions of $190 \mathrm{~nm}$ average diameter and $25 \mathrm{~nm}$ thickness (Figure 1a). To prevent oxidation of the $\mathrm{Ag}, \mathrm{Cu}$ and Pd constituents we annealed all the samples in reducing atmosphere $\left(4 \% \mathrm{H}_{2}\right.$ in $\left.\mathrm{Ar}\right)$ at $773 \mathrm{~K}$ for $24 \mathrm{~h}$. To determine the constituent layer thicknesses for the intended alloy compositions we use the reported taper angle of $60^{\circ}$ for nanostructures grown by using HCL-fabricated evaporation masks (see SI). ${ }^{26,28}$ After annealing, we measured the composition of the alloy nanodisks by energy-dispersive $\mathrm{X}$-ray spectroscopy in a scanning electron microscope (SEM/EDS, see Methods section for details) and found all of them to match the nominal compositions with astonishing precision (Figure 1b). It is therefore motivated to highlight the achieved precision and put it in perspective.

Common colloidal synthesis methods for alloy nanoparticles rely on the mixing of appropriate amounts of the alloy precursor compounds, which is limited in accuracy by the "human factor". Furthermore, colloidal synthesis often requires physical transfer of materials from one test tube to another. These two factors cause significant day-to-day uncertainties and thus negatively affect both batch-to-batch reproducibility and overall accuracy of alloy composition. These issues are surpassed using our nanolithography-based approach, where the deposition of each metallic layer is carried out in a state-of-the-art electron-beam evaporation system with thickness accuracy and reproducibility at the Ångström level. This aspect, together with the fact that we assemble the alloy nanoparticles directly on the final support, promises highly reproducible fabrication with clear advantages over colloidal synthesis in terms of composition accuracy. 
To further assess our fabrication method and analyze the spatial homogeneity of the alloys, we performed EDS elemental mapping of all three AuAg, AuPd and AuCu systems with 50:50 composition at the single nanoparticle level in a scanning transmission electron microscope (STEM/EDS, Figure 1c-e, respectively; for the same analysis of AuAg 10:90 and 90:10 composition see Figure S3). For all three systems we observe a homogeneous distribution of the elements throughout the whole particle, i.e. a fully homogeneous alloy is formed, in agreement with the equilibrium phase diagrams for these binary systems. ${ }^{29}$ Line-scans across the particles further confirm the alloy homogeneity for all three systems. Furthermore, we performed selected area electron diffraction (SAED) on single alloy nanoparticles for all three systems (Figure 2). Together with high-angular annular dark field (HAADF) STEM images also shown in Figure 1, this analysis reveals the polycrystalline nature of the structures. Moreover, the initial disk-shape is quite nicely retained, despite the high temperature annealing (see SI). This has important implications since it shows that most likely also geometrically more complex structures (as e.g. fabricated by EBL) could retain their target shape after post-fabrication annealing.

We now turn to an analysis of plasmonic properties, after having established the chemical homogeneity of the alloy nanoparticles formed and confirmed their structural integrity and crystallinity (Figure 3). For the AuAg alloy system, we find an orderly and smooth spectral transition of the plasmon resonance when increasing the Au content in the alloy in 10 at. \% increments from pure Ag to pure Au (Figure 3a). The resonance peak shifts around $0.2 \mathrm{eV}$ to the red (lower photon energy/longer wavelengths) from pure Ag to pure Au. The peak shifts scales monotonically with the mixing ratio, however, not in a perfectly linear fashion, in agreement with earlier work ${ }^{34,35}$ but slightly opposing to also reported linear correlations ${ }^{14,15}$ (Figure 3d). 
In the case of $\mathrm{AuCu}$, we first observe a slight increase of the plasmon energy up to 35 at. \% $\mathrm{Au}$, where it reaches a maximum of ca. $2 \mathrm{eV}$, followed by a linear decrease towards the pure $\mathrm{Au}$ value at ca. $1.9 \mathrm{eV}$. (Figure $3 \mathrm{~b}$ and d). The total peak shift observed between pure $\mathrm{Cu}$ and pure $\mathrm{Au}$ is thus significantly smaller compared to the AgAu system, in agreement with earlier reports from colloidally synthesized AuCu nanoparticles. ${ }^{36-38}$

For our last binary alloy system, AuPd, the presented detailed and systematic series of alloycomposition-dependent plasmonic properties is, to the best of our knowledge, the first of its kind. We find that adding only a small amount of Au to Pd already significantly shifts the resonance peak to the red, ca. $0.35 \mathrm{eV}$, reaching a maximum at ca. 40 at. \% Au, after which the peak position remains more or less constant and quite close to the one for pure Au (Figure 3c and d).

It is now interesting to also look at the linewidth (Figure 3e), or full-width-at-half-maximum, of the resonance peak, since it is inversely proportional to the plasmon lifetime and thus governed by the losses in the system. ${ }^{39}$ We determine its value from the extinction spectra by multiplying the peak half-width, as measured from the resonance maximum toward the low energy side, with a factor of 2, to avoid convolution with, e.g., interband absorption contributions or higher order plasmonic modes. ${ }^{39}$ For the AgAu system, we observe a monotonic decrease of the linewidth for increasing $\mathrm{Au}$ content in the alloy. For the AuCu system, the trend is almost opposite, i.e. the linewidth increases from ca. $0.3 \mathrm{eV}$ to a maximum around $0.45 \mathrm{eV}$ for a 50 at. \% Au content, before slightly decreasing towards pure Au. Finally, for the AuPd case the situation is most interesting. We first observe that, even for increasing Au content up to ca. 40 at. \%, the linewidth is Pd-like, i.e. much larger than for Au due to the additional interband damping in $\mathrm{Pd}^{39,40}$ At the 
same time, as noted above, in this regime the peak position is drastically changing and rapidly approaching the value for pure Au. Surprisingly, upon further increasing the Au content the linewidth actually further increases until it reaches a maximum at ca. 60 at. \% Au, after which it rapidly drops towards the value for pure Au. This means that the two parameters, i.e. peak position and linewidth, behave completely opposite also in this regime since above 40 at. \% Au the former is basically constant, whereas the latter changes significantly. This implies that for the AuPd alloy system, the two parameters change quite independently from each other. While elucidating the physics of this effect in detail is beyond the scope of this paper, we speculate that it could be connected to the relative contributions of the constituents to the real and imaginary parts of the complex dielectric response function of the alloy becoming significant at different compositions. This conceptual picture is based on the (slightly simplified) view that the real part of the dielectric function predominantly determines the resonance frequency of the plasmonic excitation, whereas the imaginary part accounts for the damping/losses of the system reflected in the linewidth.

As the next example for the new possibilities offered by our nanofabrication approach, we discuss a ternary alloy system, i.e. an array of 33:34:33 at. \% AuAgPd alloy nanodisks with nominal dimensions of $190 \mathrm{~nm}$ diameter and $25 \mathrm{~nm}$ thickness. As previously discussed, the fabrication of a ternary alloy requires simply the addition of a third constituent layer during evaporation. As before, we analyze the obtained alloy nanoparticles by SEM/EDS and find consistently excellent agreement between the targeted and obtained elemental composition, as shown in Figure 4a. Specifically, we measured Au, Ag and Pd concentrations of 33.2, 32.8 and 34.0 at. \%, respectively, as the average over five measurements with a standard deviation of less 
than 0.5 at. \% for all elements (see Table S2 in SI). As before, we also carried out STEM/EDS elemental mapping of a single ternary alloy nanoparticle and find again remarkably homogeneous distribution of the elements throughout the nanoparticle, indicating the formation of a homogeneous alloy also in this case (Figure 4b). The line scan across the particle (dashed red line in Figure 4b) further confirms a homogeneous distribution of the constituents. Lastly, the optical extinction spectrum of the ternary AuAgPd alloy nanoparticle array is shown in Figure 4d. Interestingly, as seen above for the AuPd binary system, the spectral linewidth is dominated by the Pd constituent in the alloy, despite only $33 \%$ abundance.

In order to illustrate further possibilities and effects becoming available with our method, we also fabricated a sample consisting of two miscible and one immiscible elemental component, i.e. Au, Pd and $\mathrm{Zn}$. As shown in Figure 5, we can form structures that consist of a homogeneously alloyed part of the two miscible components (AuPd) and a phase-segregated part of the pure immiscible component (Zn), similar to the recently reported immiscible system of AuNi. ${ }^{27}$ This finding highlights further possibilities offered by our method, i.e. the nanofabrication of arrays of bimetallic (alloy) nanostructures based on phase segregation. While this is not demonstrated here, we also anticipate the possibility to obtain core-shell nanostructures by optimizing annealing conditions.

Having established our new process of homogeneous binary and ternary plasmonic nanoparticle array nanofabrication, we now turn to a second aspect, namely the possibility to tailor the alloy nanoparticle dimensions. For this purpose, we fabricated 50:50 AuAg alloy nanoparticles with different pre-anneal diameters of 140, 170, 190 and $210 \mathrm{~nm}$, respectively, and constant thickness 
of $25 \mathrm{~nm}$, and measured their optical extinction spectra (Figure 6a). As previously reported for pure metal nanodisk arrays fabricated using the HCL method, we observe a distinct and linear spectral red-shift for increasing diameter, due to a change in nanoparticle aspect ratio in combination with radiation damping and dynamic depolarization effects ${ }^{39,40}$ (Figure 6b). This means that, also for these alloy nanoparticles, tailoring of nanoparticle size and shape can be used to control the plasmon resonance frequency across the near-visible light spectral range.

In the last part of this work, we will demonstrate a specific application of plasmonic alloy nanoparticle arrays, namely as signal transducers in plasmonic all-optical hydrogen sensing. ${ }^{41}$ The data presented here are an extension of our initial study of AuPd binary alloy nanoparticles as hysteresis-free plasmonic hydrogen sensors, ${ }^{28}$ which further significantly advances the performance. The background to these efforts is that pure Pd is a widely used transducer material in hydrogen sensor applications (not only optical) as it readily and selectively dissociates and absorbs hydrogen even at room temperature, and accordingly transforms to a hydride with significantly different dielectric properties ${ }^{41}$ However, the applicability of pure Pd as transducer material in a real hydrogen sensor is very limited as it features a large response only in a very narrow hydrogen pressure range, i.e. around the hydride formation and decomposition pressure (the equilibrium "plateau”). Moreover and very critically, Pd exhibits a wide hysteresis between absorption and desorption of hydrogen in this regime, due to a kinetic barrier induced by lattice strain upon hydride formation (decomposition) due to volume increase (decrease) of the system. ${ }^{42}$ This makes the sensor readout ambiguous within the hysteresis pressure range since it depends on the history of the hydrogen pressure, thus drastically reducing sensor accuracy. To this end, alloying $\mathrm{Pd}$ with other metals (e.g. $\mathrm{Au}, \mathrm{Ag}$ ) is known to reduce hysteresis 
significantly. ${ }^{43,44}$ The same holds true, as we have shown in our earlier work, for nanoparticle systems where alloying with up to 25 at. \% Au significantly reduced or completely eliminated hysteresis. ${ }^{28}$ Consequently it improved plasmonic hydrogen sensor accuracy to above $5 \%$ throughout the considered 1-1000 mbar hydrogen pressure range. Thus, in this respect, these sensors already met the stringent demands set by the automotive industry for future hydrogen sensors in terms of accuracy. ${ }^{45}$

Here we set out to further improve this performance by increasing the Au content to 30 at. \%. In our evaluation, we directly compare the hydrogen sensing performance of these new AuPd 30:70 structures with the "champion" system (25 at. \% Au) of our earlier work ${ }^{28}$ (Figure 7a). As before, we use the extinction change at plasmon peak maximum, AExtinction@Peak, as the readout. Clearly, also for the AuPd 30:70 alloy, hysteresis is completely suppressed at room temperature, indicating that we are hydrogenating the system above its critical point. However, in contrast to the AuPd 25:75 case reported earlier, the AuPd 30:70 system features a perfectly monotonic response to hydrogen throughout the investigated pressure range. In other words, the typical regions associated with hydrogen sorption in metals (solid solution at low hydrogen pressures, two-phase coexistence, hydride at high hydrogen pressure) are completely absent. Moreover, the total extinction change of AuPd 30:70 as response to exposure to hydrogen gas exceeds the AuPd 25:75 system by about a factor of 2.5 .

High resolution TEM (HRTEM) further confirms the polycrystalline nature of the nanoparticles (Figure 7b, left). Moreover, it is clear that the nanoparticles retain their metallic character thanks to the reducing nature of the annealing environment, as they do not show the presence of an 
oxide shell (Figure 7b, right). From the fast Fourier transform (FFT) analysis of a selected area to the left of the grain boundary (Figure 7c), two reflections are visible corresponding to a distance of $2.32 \AA$ between atomic planes. The latter falls between the values found in the literature for the (111) reflections family of Pd and $\mathrm{Au}\left(\mathrm{d}_{111, \mathrm{Pd}}=2.28 \AA\right.$ and $\mathrm{d}_{111, \mathrm{Au}}=2.35 \AA$, respectively). ${ }^{46,47}$ Yet more detailed information can be obtained from the FFT of a selected area to the right of the grain boundary (Figure 7d). The diffractogram is compatible with a FCC crystal - as expected from a PdAu alloy - with [101] as the zone axis. Moreover, it shows reflections corresponding to a distance between the atomic planes of $1.96 \AA$, which is between the values found in the literature for the (020) family of reflections of Pd and Au ( $\mathrm{d}_{020, \mathrm{Pd}}=1.94 \AA$ and $\mathrm{d}_{020, \mathrm{Au}}=2.04 \AA$, respectively). For both families of reflections, the relative difference is around $4 \%$ (corresponding to the difference in unit cell parameter). Such a difference would easily be distinguishable in FFT of lattice fringe images acquired at the present magnification. The fact that a single value of the unit cell parameter can be retrieved from the measured lattice spacings indicates a homogeneous crystal structure and unit cell size throughout the thickness of the nanodisk.

These findings have two key implications for plasmonic hydrogen sensing: (i) Optimizing alloy composition in nanoparticles provides a powerful strategy to engineer and eliminate hysteresis and thus hydrogen sensor accuracy. (ii) Optimizing alloy composition also provides a means to tailor and maximize optical contrast upon hydrogen sorption and thus optimize hydrogen sensor resolution. This, in turn, paves the way for the development of highly sensitive optical hydrogen sensors, which is one important step towards the realization of a hydrogen economy and crucial for a large-scale market introduction of hydrogen fuel cell powered vehicles. ${ }^{45}$ 


\section{CONCLUSIONS}

In conclusion we have presented a versatile bottom-up nanofabrication approach for large-area

$\left(\mathrm{cm}^{2}\right)$ metal alloy nanoparticle arrays based on successive fine-tuned deposition of alloy element layers through a nanofabricated mask, followed by thermal annealing to promote alloy formation. The approach is generic as it allows numerous alloy elements to be mixed, with excellent control over their concentration, as we showed specifically on the example of arrays of binary AuAg, AuCu and AuPd homogeneous alloy nanoparticles, as well a homogeneous ternary AuAgPd alloy. Moreover, the approach is readily extendable to other nanolithography methods (e.g. electron beam lithography as opposed to Hole-Mask Colloidal Lithography we used here) and thus allows highly controlled fabrication of alloy nanostructures in terms of their size, shape, and arrangement on the substrate. Our fabrication strategy thus provides a new means to explore alloy nanoparticles for plasmonic applications. In this field, they are of high interest because they feature the unique combination of spectrally fully tunable plasmonic characteristics that follow the same design rules as their traditional pure metal counterparts and tailored chemical properties optimized for a specific targeted function. As demonstrated on the specific example of superior plasmonic hydrogen sensing performance of an optimized AuPd alloy, this combination of tunable plasmonic and chemical properties will open the door to "alloy plasmonics", which we predict to have a significant impact in applications such as plasmonic gas sensing, plasmonic metamaterials and plasmon mediated catalysis. 


\section{METHODS}

Alloy nanoparticle array fabrication: Evaporation masks were fabricated using the standard hole-mask colloidal Lithography (HCL) process $^{26}$ on $1 \mathrm{x} 1 \mathrm{~cm}^{2}$ glass substrates (Borofloat, Schott Scandinavia $\mathrm{AB}$ ) and on TEM “windows” made in-house following the procedure by Grant et. al. ${ }^{48}$ The steps of the mask fabrication were as follows: (i) Substrates were cleaned (by ultrasonic agitation for the case of glass and only by rinsing for the TEM windows in order to not break the membrane) subsequently in acetone, isopropanol (IPA), and de-ionized (DI) water. (ii) Poly(methyl methacrylate) (PMMA, MicroChem Corporation, 4 wt \% diluted in anisole, $\mathrm{M}_{\mathrm{w}}=$ 950 000) was spin-coated onto the substrates at 2000 rpm for 1 min (yielding a PMMA thickness of 280 nm) followed by a soft baking at $443 \mathrm{~K}$ on a hotplate for $10 \mathrm{~min}$. (iii) Samples were subjected to a 5 s oxygen plasma (50 W, 250 mTorr, Plasma Therm Batchtop RIE 95m) to enhance the hydrophilicity of the sample surface. (iv) A polyelectrolyte solution (poly diallyldimethylammonium (PDDA) $\mathrm{M}_{\mathrm{W}}=200000 \div 350000$, Sigma Aldrich, 0.2 wt. \% in Milli-Q water, Millipore) was pipetted on the surface of the samples and left incubating for $40 \mathrm{~s}$ before rinsing in DI water. This created a positively charged surface layer on the PMMA surface. (v) A suspension of negatively charged polystyrene beads (PS 140 nm, 170 nm, 190 nm and 210 nm, sulfate latex, Interfacial Dynamics Corporation, 0.2 wt, \% in Milli-Q water, Millipore) was added to the surface. The size of the PS beads determines the bottom diameter of the fabricated nanodisks at the end of the processing. After 3 min incubation the suspension was rinsed away using DI water, and the samples were blown dry with nitrogen. (vi) A $15 \mathrm{~nm}$ thick Cr film was evaporated onto the surface of the samples using a Lesker PVD 225 Evaporator at a base

pressure of $5 \times 10^{-7}$ Torr, evaporation rate $1 \AA \mathrm{s}^{-1}$. For the fabrication of nanoellipses, the 
deposition was done at $45^{\circ}$ tilt angle. (vii) The PS beads were removed by tape stripping (SWT10, Nitto Scandinavia AB) for glass samples and by a wet tissue for TEM windows. This left a Cr film with holes at the positions of the stripped PS beads. (viii) The samples were subjected to 5 min oxygen plasma treatment (50 W, 250 mTorr, Plasma Therm Batchtop RIE 95m) to etch through the PMMA layer exposed beneath the holes in the Cr mask. (ix) Au and alloy elements were deposited in sequence through the mask at deposition rate of $\left.1 \AA \mathrm{s}^{-1}\right)$. The thicknesses of each individual layer determined the final composition of the alloy particles, see SI. (x) The remaining PMMA layer was dissolved in acetone, removing the mask from the sample, only leaving the nanodisk structures on the substrate after lift-off. (xi) Samples were soaked in IPA and blown dry with nitrogen. (xii) The samples were annealed in a homemade flow furnace under $4 \% \mathrm{H}_{2}$ in $\mathrm{Ar}\left(100 \mathrm{ml} \mathrm{min}^{-1}\right)$ at $773 \mathrm{~K}$ for $24 \mathrm{~h}$ to induce alloying.

Electron microscopy characterization: To facilitate the elemental analysis, nanoparticles were fabricated on the TEM windows mentioned in the previous section. The composition of the alloys was measured using an Inspect S SEM (FEI), at an acceleration voltage of $10 \mathrm{kV}$. At least three spectra were acquired for each sample. The relative composition of the nanoparticles (in wt. \% units) for a given specimen was determined by fitting each EDS spectrum using the software AZtec v. 2.4 (Oxford Instruments), and by averaging the obtained values for each metal. The relative atomic concentration was then obtained from the weight concentration using the respective tabulated atomic weight. Average and standard deviation of the atomic concentration were obtained using standard error propagation theory. STEM/EDS characterization was carried out in a FEI Titan 80-300 microscope at $120 \mathrm{kV}$ acceleration voltage. EDS mapping was performed using an INCA x-sight detector (Oxford Instruments), 
using a sampling distance of $5 \mathrm{~nm}$ and an integration time of $3 \mathrm{~s}$ for each spectrum. The spectra were fitted using the TEM Imaging and Analysis software v. 4.2 (FEI). Bright field TEM images and SAD patterns were acquired in a Tecnai T20 $\mathrm{G}^{2}$ microscope (FEI) at $200 \mathrm{kV}$ accelerating voltage. High resolution bright field TEM images were acquired using a Titan 80-300 TEM (FEI), operated at $300 \mathrm{kV}$ accelerating voltage with a post objective lens spherical aberration corrector (CETCOR unit, CEOS).

Hydrogen sensing: The hydrogen sensing experiments were carried out in a homemade vacuum chamber with optical access. The hydrogen pressure was monitored using a capacitive pressure gauge (MKS Baratron Capacitance Manometer). The temperature of the sample was measured using a thermocouple in direct contact with the sample surface and controlled by a temperature controller (Eurotherm 3216N) and a heating coil in a feedback loop manner. Throughout the hydrogenation measurements, the temperature was kept constant at $303 \mathrm{~K}$. Simultaneously, the optical transmittance through the sample was measured via two UHV-compatible sapphire windows by utilizing a fiber-coupled unpolarized white light source (Avantes AvaLight-Hal) and a fixed grating fiber coupled spectrometer (Avantes SensLine AvaSpec-2048XL). Isotherms were constructed by plotting the LSPR descriptors (i.e. peak wavelength and extinction@peak) of the sample directly during the measurement, derived by fitting of the LSPR peak in the optical spectra using a Lorentzian function. 


\section{FIGURES}

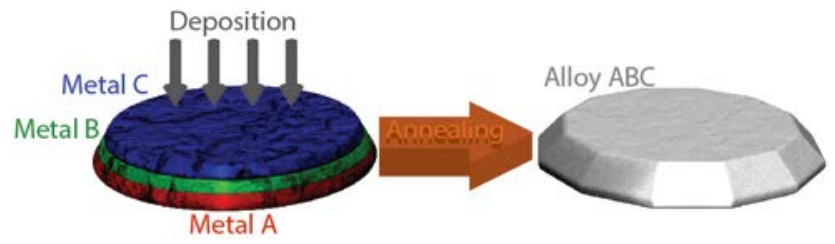

Scheme 1. Conceptual illustration of the alloy nanoparticle nanofabrication strategy. First, subsequent vapor deposition of metallic alloy component thin films is carried out through a nanofabricated mask on a substrate. The alloy proportion is determined by the thickness of the layers in conjunction with the overall dimension of the nanoparticle. Conceptually there is no limitation on the number of alloy components that can be used. After the deposition of the desired alloy component layers, thermal annealing is employed to induce alloy formation. A slight change in nanoparticle dimensions and promotion of recrystallization and grain growth in the nanoparticles is to be expected during annealing. 

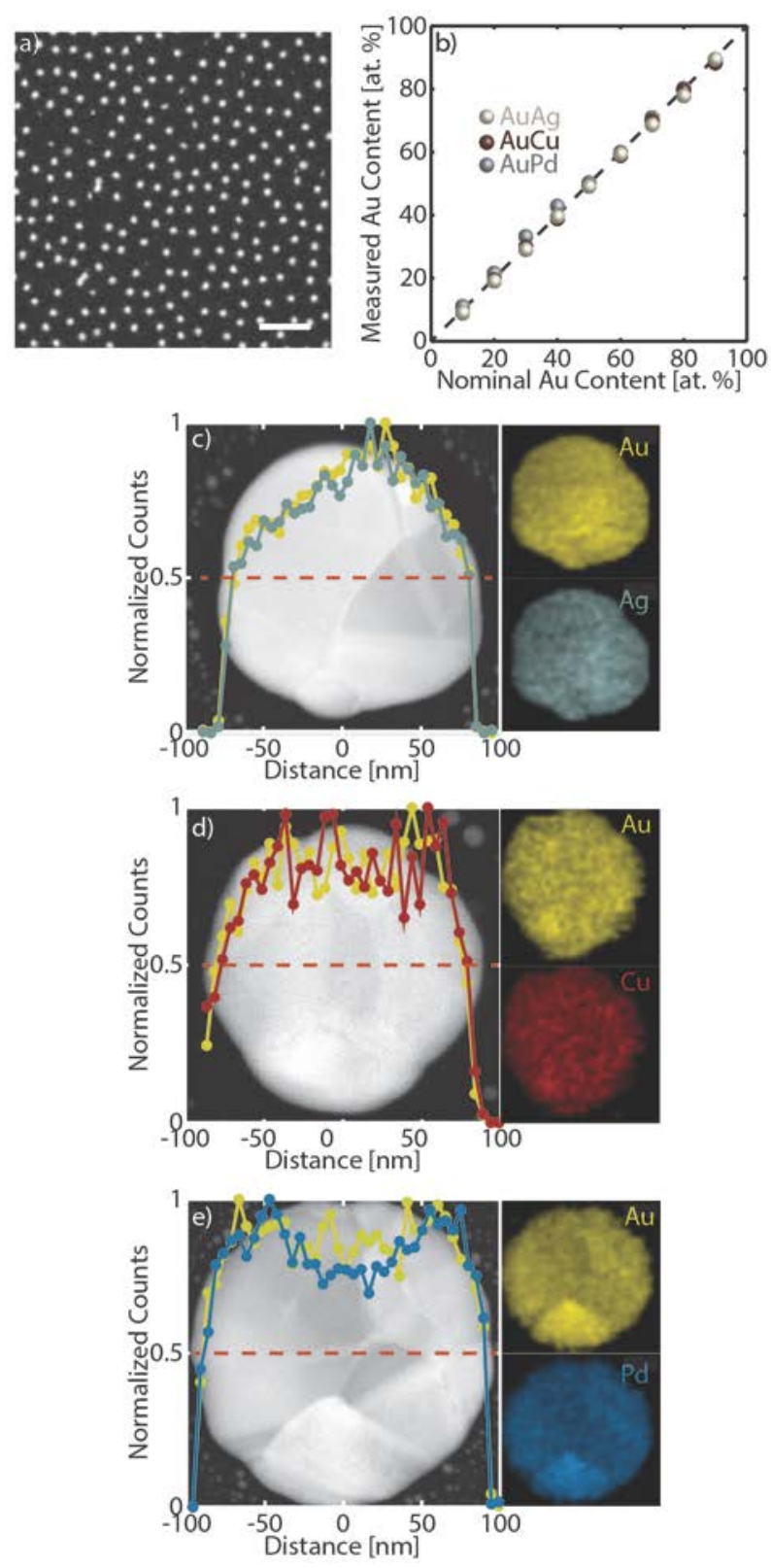

Figure 1. Binary alloy characterization. (a) SEM overview micrograph of an array of 50:50 AuAg alloy nanoparticles after annealing, scale bar: $2 \mu \mathrm{m}$. (b) The Au content determined by SEM/EDS in AuAg, AuCu and AuPd alloy nanoparticle arrays plotted vs. the nominal Au content. Note the excellent agreement that corroborates the remarkable precision of our fabrication approach. The data points are averaged over at least three measurements and error bars are smaller than the symbols, i.e. smaller than 2.5 at. \% (see Table S1 in the SI). Below, 
STEM/EDS elemental maps of single (c) AuAg, (d) AuCu and (e) AuPd alloy nanoparticles are shown in the right panels and reveal homogeneous distribution of the alloy constituents throughout the entire particle. In the left panels, high-angular annular dark field (HAADF) STEM images of representative single alloy nanoparticles are shown together with a corresponding elemental profile acquired across the particles (red dashed lines). For all systems the ratio of alloy constituents is remarkably constant throughout the entire particle, further confirming the homogeneous nature of the formed alloys. The differences in counts of both alloy elements across the particle are likely originating fro the three-dimensional topography of the nanoparticle. 

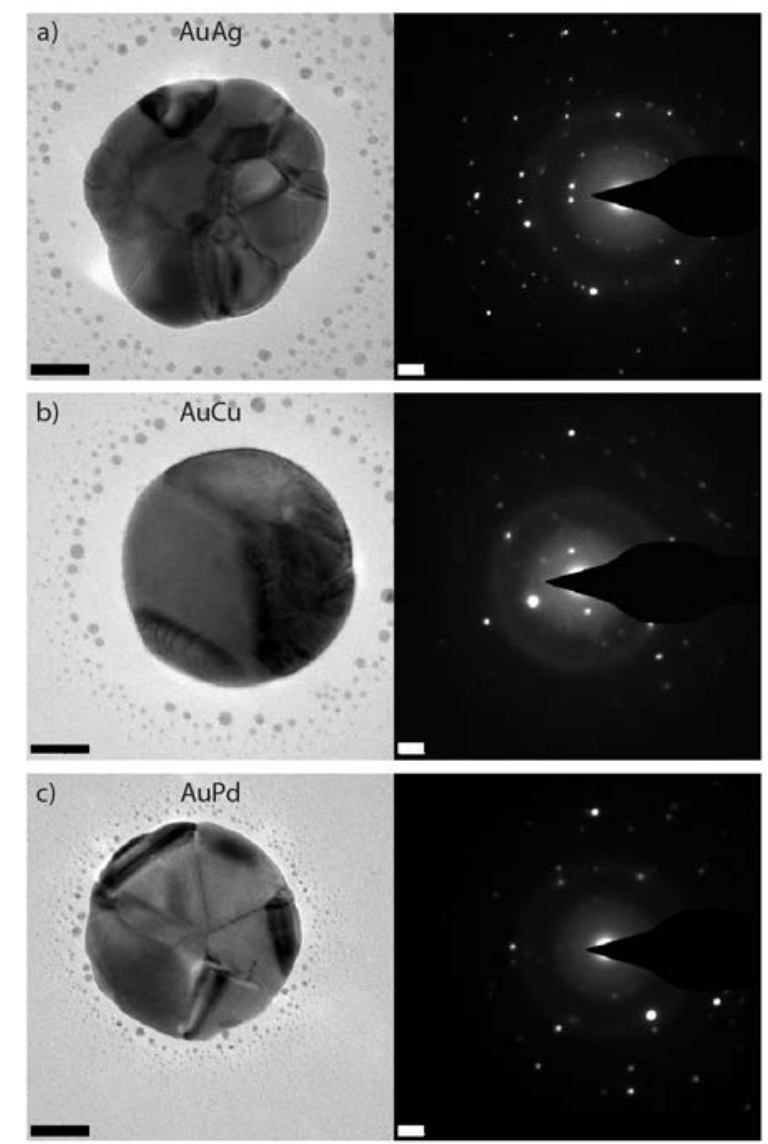

Figure 2. Crystallinity of the fabricated alloys. Left: bright field TEM images of single (a) AuAg, (b) AuCu and (c) AuPd alloy nanoparticles (scale bar $50 \mathrm{~nm}$ ). Several grains are clearly visible in each nanoparticles, indicating their polycrystalline nature. The variation in diameter of the nanoparticles is most likely due to the polydispersity of the polystyrene beads used during the mask fabrication process, as discussed in the SI and the corresponding Figure S4. Right: corresponding SAD patterns (right, scale bar $2 \mathrm{~nm}^{-1}$ ). The polycrystalline nature of the nanoparticles is further confirmed. The halo of small particles present around the nanodisks is a feature inherent to nanostructures fabricated using a lithography mask with an undercut. Its origin is deflection of incoming evaporated species from the mask rim such that they land "off target” and thus form the halo. 

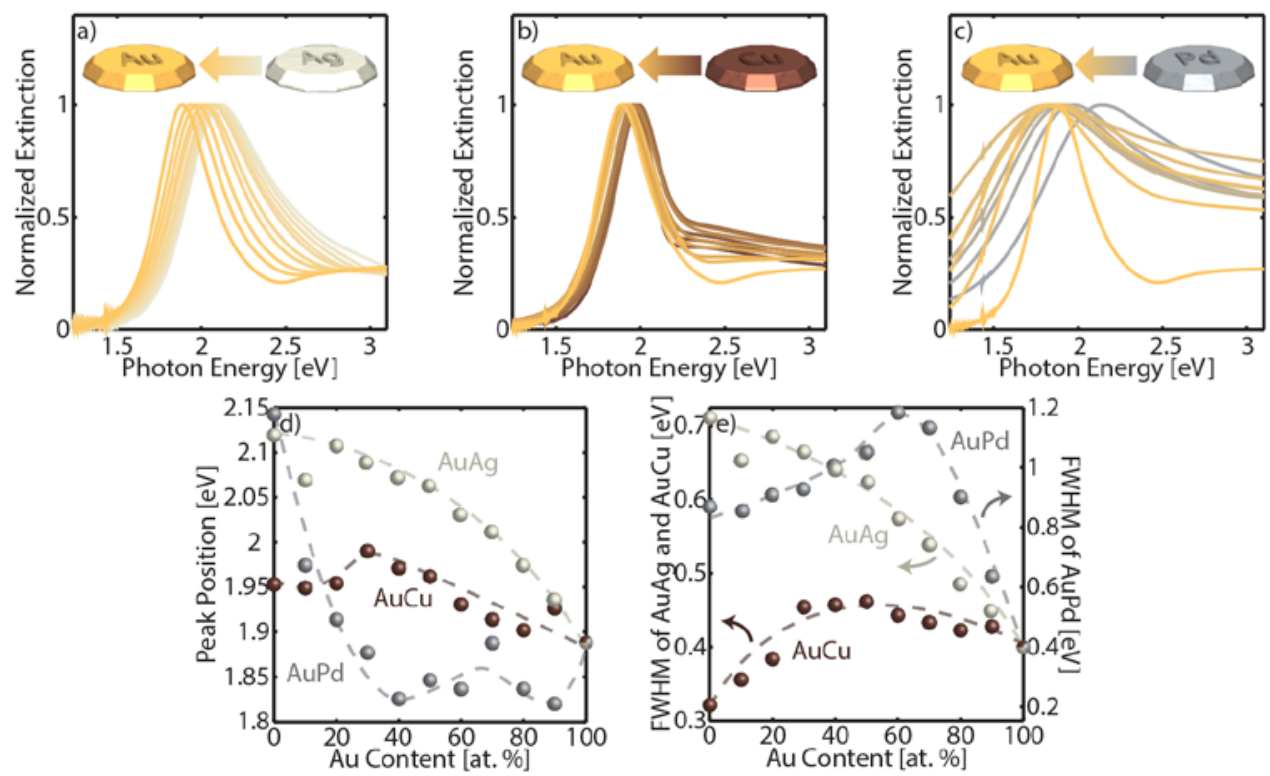

Figure 3. Plasmonic response of binary allos nanoparticle arrays. The evolution of normalized extinction spectra for (a) AuAg, (b) AuCu and (c) AuPd alloy systems for systematically increasing $\mathrm{Au}$ atomic content (0-100 at. \%, in steps of 10 at. \%) and their extracted (d) resonance peak position and (e) spectral linewidth. For AuAg, the extinction spectra transition smoothly between their pure constituent counterparts, as clearly seen from their resonancepeak position position (d) and linewidth (e) values. In the case of $\mathrm{AuCu}$ and AuPd, two regimes can be noticed for both peak position and linewidth. For the AuCu system, a constant peak position is observed for low Au content up to 30 at.\% followed by a decrease towards pure Au, while the opposite trend is observed for the linewidth. Similar trends are also seen for AuPd alloys where the peak position changes significantly for low Au content up to 40 at. \% before it becomes more or less constant, whereas for the linewidth basically the opposite behavior is observed. 

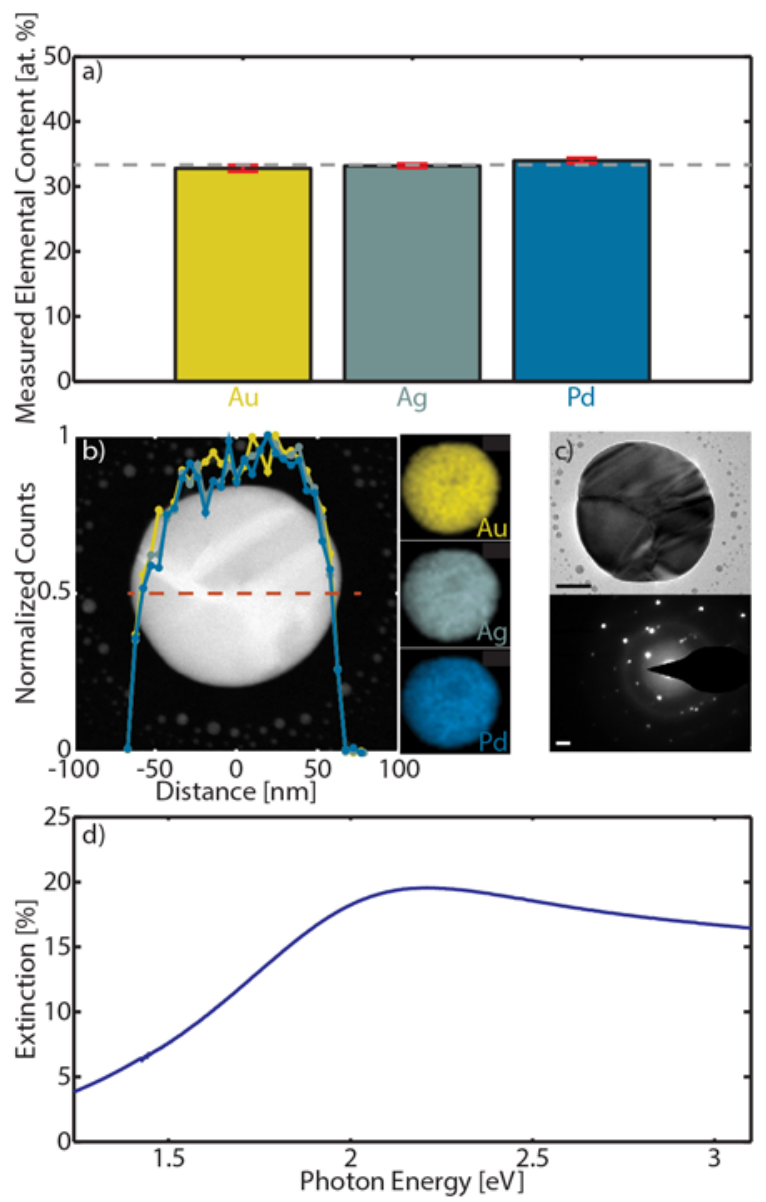

Figure 4. Ternary AuAgPd alloy characterization. (a) The measured Au (yellow), Ag (grey) and Pd (blue) content in nominal 33:34:33 AuAgPd ternary alloy, as derived from SEM/EDS analysis. Note the excellent agreement between nominal and measured composition. The dashed line marks 33.33 at. \% and the error bars (red lines, less than 0.5 at. \%) represent the standard deviation over five measurements (see Table S2 in the SI). (b) Elemental mapping of AuAgPd. The right panels show elemental maps of a single AuAgPd particle for Au (yellow), Ag (grey) and Pd (blue). In the left panel a high-angular annular dark field (HAADF) STEM image together with a corresponding elemental profile acquired across the particle (red dashed line) further confirms the homogeneous constituent distribution. (c) Bright field TEM image of a nanoparticle (top, scale bar $50 \mathrm{~nm}$ ) and corresponding SAD pattern (bottom, scale bar $2 \mathrm{~nm}^{-1}$ ) 
confirming the polycrystalline nature of the structure. (d) Optical extinction spectrum of a quasirandom array of AuAgPd nanoparticles. We note again the dominant effect of Pd (despite only 33\% abundance) manifested by the peak linewidth, which is much larger than for Au and Ag nanoparticles. 


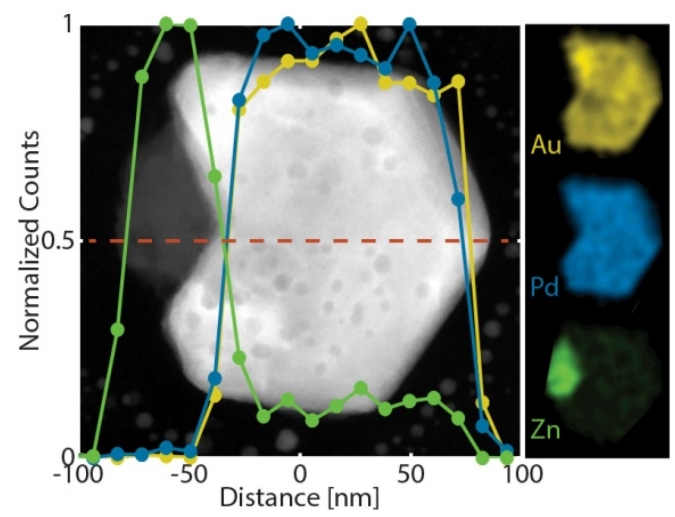

Figure 5. Combining phase-segregation and alloying in the AuAgZn system. The figure shows a STEM/EDS elemental map (right panels) together with a (HAADF) STEM image and the corresponding elemental profile acquired across the particle (red dashed line). As can be seen, when combining two miscible components (AuPd) and an immiscible component ( $\mathrm{Zn}$ ), both alloying (between Au and Pd) and segregation of Zn occur during annealing. This opens up intriguing possibilities for the fabrication of heterostructures consisting of alloy and pure element components in the same nanostructure. 

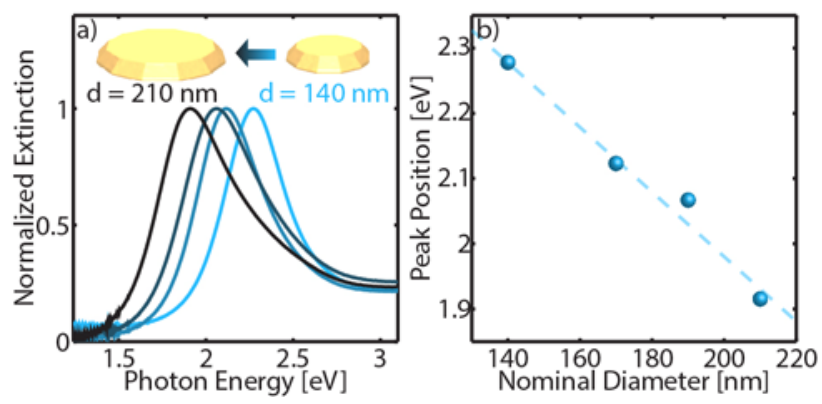

Figure 6. Spectral tunability of alloy nanoparticles by dimension design. (a) The normalized optical extinction spectra evolution of 50:50 AuAg alloy nanoparticles with different nominal diameters (140, 170, 190 and $210 \mathrm{~nm}$ ) and constant $25 \mathrm{~nm}$ nominal thickness. (b) The extracted peak positions correlate linearly with the change of the diameter of the nanoparticles, in agreement with the well-established trends found for pure noble metal plasmonic nanoparticle systems. The dashed line is guide for the eye. 

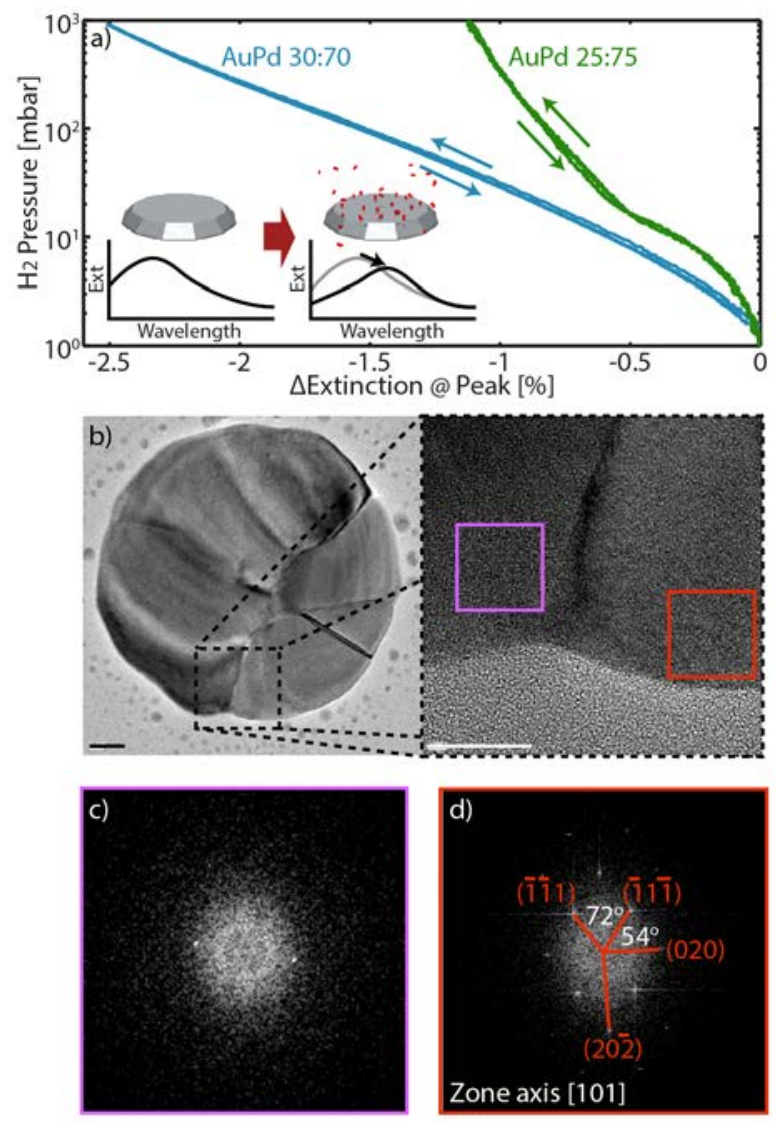

Figure 7. (a) Hydrogen absorption and desorption isotherms of AuPd 30:70 (blue) and 25:75 (green) binary alloy systems. The upward and downward arrows indicate the direction of the hydrogen pressure change (i.e. absorption and desorption, respectively). All isotherms were measured at $303 \mathrm{~K}$ by tracking the extinction change at plasmon peak maximum, AExtinction@Peak. Clearly both alloys do not feature any hysteresis. However the AuPd 30:70 alloy shows higher sensitivity and exhibits a monotonic response to changing hydrogen pressure throughout the entire 1-1000 mbar hydrogen pressure range. Inset: the change of the alloy plasmonic peak resonance upon hydrogen absorption. The AuPd 25:75 data is adopted from Wadell et al. ${ }^{28}$ (b) TEM characterization. Left: bright field image of a single AuPd 30:70 nanodisk (scale bar $20 \mathrm{~nm}$ ). Right: higher magnification image of two adjacent crystallites (scale bar $10 \mathrm{~nm}$ ). (c) FFT of a selected area to the left of the grain boundary in panel b). Two 
reflections are visible, corresponding to a distance between the atomic planes is $2.32 \AA$, which is between the values found in the literature for the (111) family of reflections of Pd and Au ( $\mathrm{d}_{111, \mathrm{Pd}}$ $=2.28 \AA$ and $\mathrm{d}_{111, \mathrm{Au}}=2.35 \AA$, respectively). ${ }^{46,47}$ (d) FFT of a selected area to the right of the grain boundary in panel b). The diffractogram is characteristic of a FCC crystal with [101] as the zone axis. The reflections corresponding to the families of planes (020), (111) and (202) can be identified, with respective interplanar distances $\mathrm{d}_{020}=1.96 \AA, \mathrm{d}_{111}=2.32 \AA$ and $\mathrm{d}_{202}=1.42 \AA$. 


\section{ASSOCIATED CONTENT}

\section{Supporting Information}

Geometrical considerations, optical spectra of unalloyed layered precursor structures, SEM/EDS analysis, TEM EDS elemental maps for AuAg of 10:90 and 90:10, SEM images of nanodisk and nanoellipse arrays before and after annealing, and hydrogen sensing response expressed in the peak position change $\left(\Delta \lambda_{\text {peak }}\right)$. This material is available free of charge via the Internet at http://pubs.acs.org.

\section{AUTHOR INFORMATION}

\section{Corresponding Author}

*(FAAN): ferryn@chalmers.se (CL): clangham@chalmers.se

\section{Author Contributions}

The manuscript was written through contributions of all authors. All authors have given approval to the final version of the manuscript. $¥$ These authors contributed equally.

\section{ACKNOWLEDGMENTS}

We acknowledge financial support from the Swedish Foundation for Strategic Research Framework Program RMA11-0037 (FAAN \& CL), the ERC Starting Grant project SINCAT (CL), the Swedish Research Council (CL), and the Knut and Alice Wallenberg Stiftelse for their support of the $\mu$-fab cleanroom infrastructure in Sweden. The research leading to these results has also received funding from the People Programme (Marie Curie Actions) of the European 
Union's Seventh Framework Programme (FP7/2007-2013) under REA grant agreement $\mathrm{n}^{\circ}$ 609405 (COFUNDPostdocDTU) (BI). 


\section{REFERENCES}

(1) Wang, D.; Li, Y. Bimetallic Nanocrystals: Liquid-Phase Synthesis and Catalytic Applications. Adv. Mater. 2011, 23, 1044-1060.

(2) Liu, X.; Wang, D.; Li, Y. Synthesis and Catalytic Properties of Bimetallic Nanomaterials with Various Architectures. Nano Today 2012, 7, 448-466.

(3) Singh, A. K.; Xu, Q. Synergistic Catalysis over Bimetallic Alloy Nanoparticles. ChemCatChem 2013, 5, 652-676.

(4) Chen, G.; Zhao, Y.; Fu, G.; Duchesne, P. N.; Gu, L.; Zheng, Y.; Weng, X.; Chen, M.; Zhang, P.; Pao, C.-W.; Lee, J.-F.; Zheng, N. Interfacial Effects in Iron-Nickel HydroxidePlatinum Nanoparticles Enhance Catalytic Oxidation. Science 2014, 344, 495-499.

(5) Cortie, M. B.; McDonagh, A. M. Synthesis and Optical Properties of Hybrid and Alloy Plasmonic Nanoparticles. Chem. Rev. 2011, 111, 3713-3735.

(6) Blaber, M. G.; Arnold, M. D.; Ford, M. J. A Review of the Optical Properties of Alloys and Intermetallics for Plasmonics. J. Phys. Condens. Matter 2010, 22, 143201.

(7) You, H.; Yang, S.; Ding, B.; Yang, H. Synthesis of Colloidal Metal and Metal Alloy Nanoparticles for Electrochemical Energy Applications. Chem. Soc. Rev. 2013, 42, 28802904.

(8) Sun, S. Recent Advances in Chemical Synthesis, Self-Assembly, and Applications of FePt Nanoparticles. Adv. Mater. 2006, 18, 393-403.

(9) Sra, A. K.; Schaak, R. E. Synthesis of Atomically Ordered $\mathrm{AuCu}$ and $\mathrm{AuCu}(3)$ Nanocrystals from Bimetallic Nanoparticle Precursors. J. Am. Chem. Soc. 2004, 126, 6667-6672.

(10) Chen, W.; Yu, R.; Li, L.; Wang, A.; Peng, Q.; Li, Y. A Seed-Based Diffusion Route to Monodisperse Intermetallic CuAu Nanocrystals. Angew. Chemie 2010, 122, 2979-2983.

(11) Wang, G.; Xiao, L.; Huang, B.; Ren, Z.; Tang, X.; Zhuang, L.; Lu, J. AuCu Intermetallic Nanoparticles: Surfactant-Free Synthesis and Novel Electrochemistry. J. Mater. Chem. 2012, 22, 15769-15774. 
(12) Wu, M.-L.; Chen, D.-H.; Huang, T.-C. Synthesis of Au/Pd Bimetallic Nanoparticles in Reverse Micelles. Langmuir 2001, 17, 3877-3883.

(13) Kan, C.; Cai, W.; Li, C.; Zhang, L.; Hofmeister, H. Ultrasonic Synthesis and Optical Properties of Au/Pd Bimetallic Nanoparticles in Ethylene Glycol. J. Phys. D. Appl. Phys. 2003, 36, 1609-1614.

(14) Link, S.; Wang, Z. L.; El-Sayed, M. A. Alloy Formation of Gold-Silver Nanoparticles and the Dependence of the Plasmon Absorption on Their Composition. J. Phys. Chem. B 1999, 103, 3529-3533.

(15) Mallin, M. P.; Murphy, C. J. Solution-Phase Synthesis of Sub-10 Nm Au-Ag Alloy Nanoparticles. Nano Lett. 2002, 2, 1235-1237.

(16) Zhang, Q.; Xie, J.; Liang, J.; Lee, J. Y. Synthesis of Monodisperse Ag-Au Alloy Nanoparticles with Independently Tunable Morphology, Composition, Size, and Surface Chemistry and Their 3-D Superlattices. Adv. Funct. Mater. 2009, 19, 1387-1398.

(17) Liu, S.; Chen, G.; Prasad, P. N.; Swihart, M. T. Synthesis of Monodisperse Au, Ag, and $\mathrm{Au}-\mathrm{Ag}$ Alloy Nanoparticles with Tunable Size and Surface Plasmon Resonance Frequency. Chem. Mater. 2011, 23, 4098-4101.

(18) Rioux, D.; Meunier, M. Seeded Growth Synthesis of Composition and Size-Controlled Gold-Silver Alloy Nanoparticles. J. Phys. Chem. C 2015, 119, 13160-13168.

(19) Bohren, C. F.; Huffman, D. R. Absorption and Scattering of Light by Small Particles; Wiley-VCH: New York, 2007.

(20) Langhammer, C.; Kasemo, B.; Zorić, I. Absorption and Scattering of Light by Pt, Pd, Ag, and Au Nanodisks: Absolute Cross Sections and Branching Ratios. J. Chem. Phys. 2007, 126, 194702.

(21) Zheludev, N. I.; Kivshar, Y. S. From Metamaterials to Metadevices. Nat. Mater. 2012, 11, 917-924.

(22) Gunnarsson, L.; Rindzevicius, T.; Prikulis, J.; Kasemo, B.; Käll, M.; Zou, S.; Schatz, G. C. Confined Plasmons in Nanofabricated Single Silver Particle Pairs: Experimental Observations of Strong Interparticle Interactions. J. Phys. Chem. B 2005, 109, 1079-1087. 
(23) Antosiewicz, T. J.; Apell, S. P.; Zäch, M.; Zorić, I.; Langhammer, C. Oscillatory Optical Response of an Amorphous Two-Dimensional Array of Gold Nanoparticles. Phys. Rev. Lett. 2012, 109, 247401.

(24) Liu, N.; Guo, H.; Fu, L.; Kaiser, S.; Schweizer, H.; Giessen, H. Three-Dimensional Photonic Metamaterials at Optical Frequencies. Nat. Mater. 2008, 7, 31-37.

(25) Haynes, C. L.; Van Duyne, R. P. Nanosphere Lithography: A Versatile Nanofabrication Tool for Studies of Size-Dependent Nanoparticle Optics. J. Phys. Chem. B 2001, 105, 5599-5611.

(26) Fredriksson, H.; Alaverdyan, Y.; Dmitriev, A.; Langhammer, C.; Sutherland, D. S.; Zäch, M.; Kasemo, B. Hole-Mask Colloidal Lithography. Adv. Mater. 2007, 19, 4297-4302.

(27) Chen, P.-C.; Liu, G.; Zhou, Y.; Brown, K. A.; Chernyak, N.; Hedrick, J. L.; He, S.; Xie, Z.; Lin, Q.-Y.; Dravid, V. P.; O'Neill-Slawecki, S. A.; Mirkin, C. A. Tip-Directed Synthesis of Multimetallic Nanoparticles. J. Am. Chem. Soc. 2015, 137, 9167-9173.

(28) Wadell, C.; Nugroho, F. A. A.; Lidström, E.; Iandolo, B.; Wagner, J. B.; Langhammer, C. Hysteresis-Free Nanoplasmonic Pd-Au Alloy Hydrogen Sensors. Nano Lett. 2015, 15, 3563-3570.

(29) Alloy Phase Diagrams; Baker, H., Ed.; ASM International: Materials Park, OH, 1992.

(30) Frederiksen, M.; Sutherland, D. S. Direct Modification of Colloidal Hole-Masks for Locally Ordered Hetero-Assemblies of Nanostructures over Large Areas. Nanoscale 2014, 6, 731-735.

(31) Syrenova, S.; Wadell, C.; Langhammer, C. Shrinking-Hole Colloidal Lithography: SelfAligned Nanofabrication of Complex Plasmonic Nanoantennas. Nano Lett. 2014, 14, 2655-2663.

(32) Tinguely, J.-C.; Sow, I.; Leiner, C.; Grand, J.; Hohenau, A.; Felidj, N.; Aubard, J.; Krenn, J. R. Gold Nanoparticles for Plasmonic Biosensing: The Role of Metal Crystallinity and Nanoscale Roughness. Bionanoscience 2011, 1, 128-135.

(33) Jung, Y. S.; Sun, Z.; Kim, H. K.; Blachere, J. Blueshift of Surface Plasmon Resonance Spectra in Anneal-Treated Silver Nanoslit Arrays. Appl. Phys. Lett. 2005, 87, 263116. 
(34) Treguer, M.; de Cointet, C.; Remita, H.; Khatouri, J.; Mostafavi, M.; Amblard, J.; Belloni, J.; de Keyzer, R. Dose Rate Effects on Radiolytic Synthesis of Gold-Silver Bimetallic Clusters in Solution. J. Phys. Chem. B 1998, 102, 4310-4321.

(35) Sánchez-Ramírez, J. F.; Pal, U.; Nolasco-Hernández, L.; Mendoza-Álvarez, J.; PescadorRojas, J. A. Synthesis and Optical Properties of Au-Ag Alloy Nanoclusters with Controlled Composition. J. Nanomater. 2008, 620412.

(36) Smetana, A. B.; Klabunde, K. J.; Sorensen, C. M.; Ponce, A. A.; Mwale, B. LowTemperature Metallic Alloying of Copper and Silver Nanoparticles with Gold Nanoparticles through Digestive Ripening. J. Phys. Chem. B 2006, 110, 2155-2158.

(37) Kim, M.-J.; Na, H.-J.; Lee, K. C.; Yoo, E. A.; Lee, M. Preparation and Characterization of $\mathrm{Au}-\mathrm{Ag}$ and $\mathrm{Au}-\mathrm{Cu}$ Alloy Nanoparticles in Chloroform. J. Mater. Chem. 2003, 13, 17891792.

(38) Cattaruzza, E.; Battaglin, G.; Gonella, F.; Polloni, R.; Scremin, B. F.; Mattei, G.; Mazzoldi, P.; Sada, C. Au-Cu Nanoparticles in Silica Glass as Composite Material for Photonic Applications. Appl. Surf. Sci. 2007, 254, 1017-1021.

(39) Zorić, I.; Zäch, M.; Kasemo, B.; Langhammer, C. Gold, Platinum, and Aluminum Nanodisk Plasmons: Material Independence, Subradiance, and Damping Mechanisms. ACS Nano 2011, 5, 2535-2546.

(40) Langhammer, C.; Yuan, Z.; Zorić, I.; Kasemo, B. Plasmonic Properties of Supported Pt and Pd Nanostructures. Nano Lett. 2006, 6, 833-838.

(41) Wadell, C.; Syrenova, S.; Langhammer, C. Plasmonic Hydrogen Sensing with Nanostructured Metal Hydrides. ACS Nano 2014, 8, 11925-11940.

(42) Schwarz, R. B.; Khachaturyan, A. G. Thermodynamics of Open Two-Phase Systems with Coherent Interfaces: Application to Metal-Hydrogen Systems. Acta Mater. 2006, 54, 313323.

(43) Hughes, R. C.; Schubert, W. K.; Zipperian, T. E.; Rodriguez, J. L.; Plut, T. A. Thin-Film Palladium and Silver Alloys and Layers for Metal-Insulator-Semiconductor Sensors. J. Appl. Phys. 1987, 62, 1074-1083.

(44) Hughes, R. C.; Schubert, W. K. Thin Films of Pd/Ni Alloys for Detection of High 
Hydrogen Concentrations. J. Appl. Phys. 1992, 71, 542-544.

(45) Boon-Brett, L.; Bousek, J.; Black, G.; Moretto, P.; Castello, P.; Hübert, T.; Banach, U. Identifying Performance Gaps in Hydrogen Safety Sensor Technology for Automotive and Stationary Applications. Int. J. Hydrogen Energy 2010, 35, 373-384.

(46) Hull, A. W. X-Ray Crystal Analysis of Thirteen Common Metals. Phys. Rev. 1921, 17, 571-588.

(47) Jette, E. R.; Foote, F. Precision Determination of Lattice Constants. J. Chem. Phys. 1935, 3, 605-616.

(48) Grant, A. W.; Hu, Q.-H.; Kasemo, B. Transmission Electron Microscopy Windows for Nanofabricated Structures. Nanotechnology 2004, 15, 1175-1181. 
TOC Figure

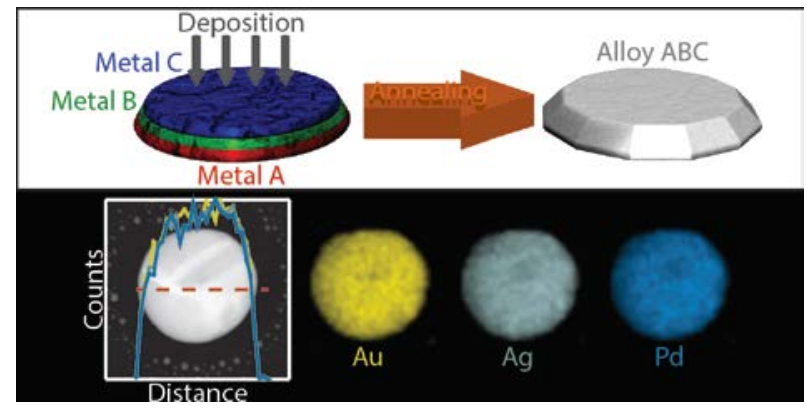




\title{
Supporting Information
}

\section{Bottom-Up Nanofabrication of Supported Noble Metal Alloy Nanoparticle Arrays for Plasmonics}

\author{
Ferry A. A. Nugroho ${ }^{*, \neq, 1}$, Beniamino Iandolo ${ }^{\ddagger 2}$, Jakob B. Wagner ${ }^{2}$ and Christoph \\ Langhammer*,1 \\ ${ }^{1}$ Department of Physics, Chalmers University of Technology, 41296 Göteborg, Sweden \\ ${ }^{2}$ Center for Electron Nanoscopy, Technical University of Denmark, 2800, Kongens Lyngby, \\ Denmark
}

Email:*(FAAN): ferryn@chalmers.se (CL): clangham@chalmers.se 


\section{Geometrical Considerations}

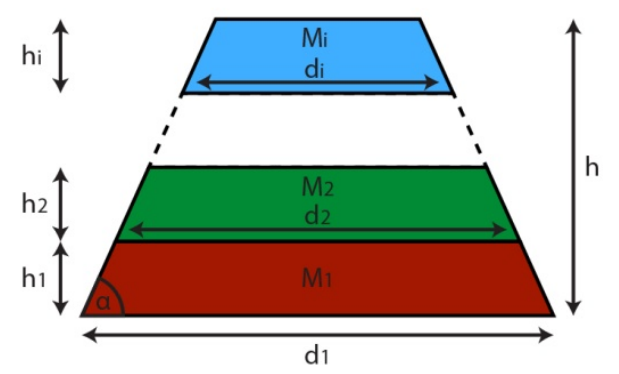

Scheme S1. The geometrical considerations for alloy composition determination for the case of a tapered cone. Each metal constituent layer $\left(M_{i}, i=1,2,3 \ldots, n\right)$ is defined by its diameter $\left(d_{i}\right)$ and height $\left(h_{i}\right)$. However, as described in the text below, the alloy proportions are dictated solely by the thickness of each layer, given the total height $(h)$, base diameter $\left(d_{1}\right)$ and taper angle $(\alpha)$.

The atomic composition of the alloy nanoparticles is controlled by varying the volume of their constituent layers. As discussed in the main text, material deposition through a mask creates a tapered structure, ${ }^{1,2}$ as sketched in Scheme S1. The volume of a tapered cone $\left(V_{\mathrm{tc}}\right)$ is known as:

$V_{\mathrm{tc}}=\frac{1}{12} \pi h_{\mathrm{tc}}\left(3 d_{t c}^{2}-\frac{6 h_{\mathrm{tc}} d_{t c}}{\tan \alpha}+\frac{4 h_{\mathrm{tc}}^{2}}{\tan ^{2} \alpha}\right)$

where $\alpha, d_{\mathrm{tc}}$ and $h_{\mathrm{tc}}$ are the cone's taper angle, diameter and height, respectively. Hence a general formula for each constituent layer with diameter $d_{i}$ and height $h_{i}$ (Scheme S1) is:

$V_{\mathrm{i}}=\frac{1}{12} \pi h_{\mathrm{i}}\left(3 d_{i}^{2}-\frac{6 h_{\mathrm{i}} d_{i}}{\tan \alpha}+\frac{4 h_{\mathrm{i}}^{2}}{\tan ^{2} \alpha}\right)$.

However, from the schematic it is clear that each constituent diameter is related to the height and the diameter of the prior constituent(s) as

$d_{\mathrm{i}}=d_{\mathrm{i}-1}-2 \frac{h_{i-1}}{\tan \alpha}$

which in the end can be expressed as a function of the base diameter $d_{1}$

$$
d_{\mathrm{i}}=d_{1}-2 \frac{\left(h_{i-1}+h_{i-2}+\cdots+h_{1}\right)}{\tan \alpha} .
$$

Furthermore, the height of each constituent layer $h_{i}$ is related to each other as:

$h_{1}+h_{2}+\cdots+h_{\mathrm{i}}=h$

By substituting equation 4 and 5 into equation 2, the layer volume can be expressed as a function of taper angle, base diameter and total height. 
Knowing the volume of the respective constituent layers, it is then straightforward to define the number of atoms in each layer (in other words, the number of atoms for each metal) as:

$n_{\mathrm{i}}=\frac{\rho_{i} V_{i}}{M_{i}} N_{A}$

where $\rho, M$, and $N_{A}$ are the density, molar mass and Avogadro number, respectively.

And lastly their atomic percentage can be determined,

at. $\%_{i}=\frac{n_{i}}{\sum_{j=1}^{n} n_{j}}$

For our work, we fabricated the alloy nanoparticles with $190 \mathrm{~nm}$ diameter and $25 \mathrm{~nm}$ height by depositing the Au layer first. The angle $\alpha$ will be material dependent. In our case, we have estimated $\alpha$ to be $\sim 60^{\circ}$ for previous work. ${ }^{3}$ Knowing all these parameters, we can plot the $\mathrm{Au}$ thickness required for any desired $\mathrm{AuAg}, \mathrm{AuCu}$ and AuPd compositions, as shown in Figure S1.

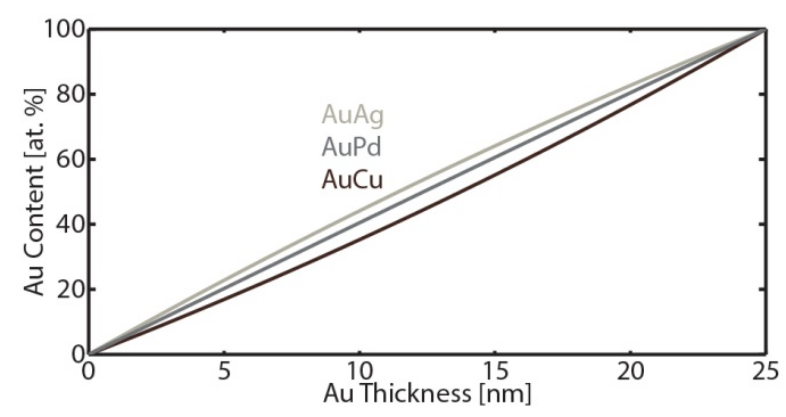

Figure S1. Au content for different Au layer thicknesses in binary alloy systems $A u A g, A u C u$ and AuPd with $190 \mathrm{~nm}$ diameter, $25 \mathrm{~nm}$ height and $60^{\circ}$ taper angle. The relation holds true if the Au layer is deposited first. 


\section{Plasmonic Response of Layered Nanostructures Prior to Annealing}
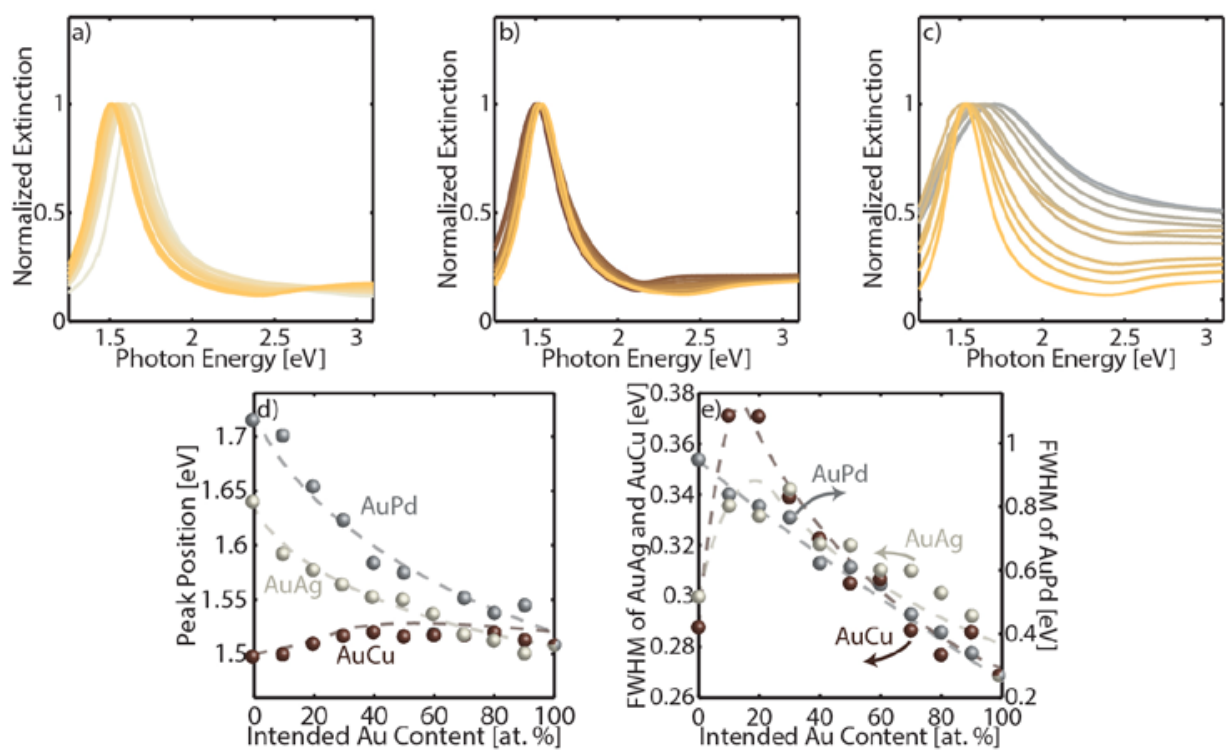

Figure S2. Plasmonic response of unalloyed layered of (a) AuAg, (b) AuCu and (c) AuPd nanodisks with different layer thicknesses for the intended alloy compositions (cf. Figure S1).

\section{SEM/EDS Analysis}

Table S1. SEM/EDS analysis of the binary alloy AuAg, AuCu and AuPd compositions.

\begin{tabular}{|c|c|c|c|}
\hline \multirow{2}{*}{$\begin{array}{c}\text { Nominal } \\
\text { Au Content (at. \%) }\end{array}$} & \multicolumn{3}{|c|}{ Measured Au Content (at. \%) } \\
\cline { 2 - 4 } & AuAg & AuCu & AuPd \\
\hline 10 & $9.0 \pm 0.53$ & $8.93 \pm 0.22$ & $11.09 \pm 0.69$ \\
\hline 20 & $19.0 \pm 0.65$ & $19.47 \pm 1.30$ & $21.62 \pm 1.06$ \\
\hline 30 & $29.03 \pm 0.18$ & $29.94 \pm 0.80$ & $33.25 \pm 0.36$ \\
\hline 40 & $39.53 \pm 1.28$ & $38.89 \pm 0.36$ & $42.85 \pm 0.35$ \\
\hline 50 & $49.1 \pm 0.72$ & $49.22 \pm 0.53$ & $50.25 \pm 0.15$ \\
\hline 60 & $59.36 \pm 0.66$ & $58.95 \pm 0.78$ & $59.96 \pm 0.61$ \\
\hline 70 & $68.64 \pm 0.51$ & $69.85 \pm 2.42$ & $70.94 \pm 0.49$ \\
\hline 80 & $77.81 \pm 0.23$ & $79.62 \pm 0.93$ & $80.23 \pm 0.30$ \\
\hline 90 & $89.48 \pm 0.61$ & $88.28 \pm 1.31$ & $89.43 \pm 0.11$ \\
\hline
\end{tabular}

Table S2. SEM/EDS analysis of the ternary alloy AuAgPd composition.

\begin{tabular}{|c|c|c|c|c|c|}
\hline \multicolumn{3}{|c|}{ Nominal Elemental Content (at. \%) } & \multicolumn{3}{c|}{ Measured Elemental Content (at. \%) } \\
\hline $\mathrm{Au}$ & $\mathrm{Ag}$ & $\mathrm{Pd}$ & $\mathrm{Au}$ & $\mathrm{Ag}$ & $\mathrm{Pd}$ \\
\hline 33 & 34 & 33 & $32.80 \pm 0.50$ & $33.20 \pm 0.30$ & $34.00 \pm 0.40$ \\
\hline
\end{tabular}




\section{TEM EDS Elemental Maps of AuAg 10:90 and 90:10 Alloy}
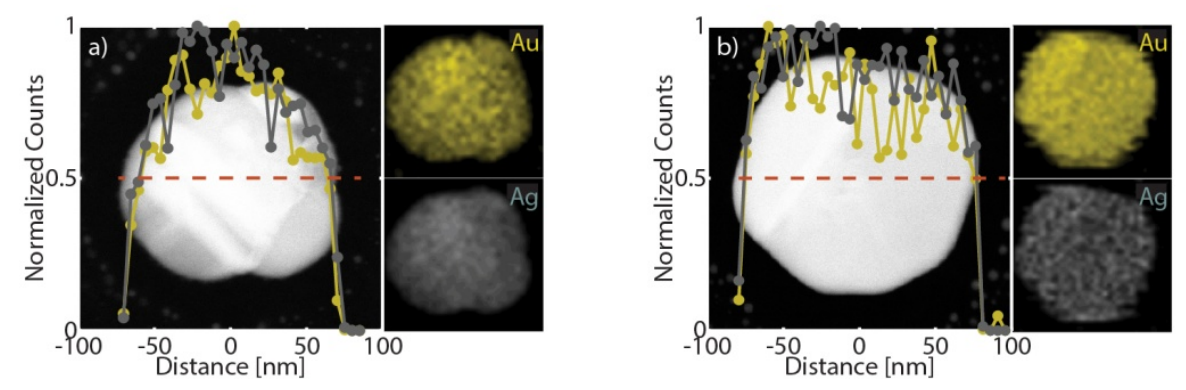

Figure S3. EDS elemental maps of AuAg of (a) 10:90 and (b) 90:10 composition, respectively. The right panels reveal homogenous distribution of both alloy constituents throughout the particles. The left panels show high-angular annular dark field (HAADF) STEM images of representative single alloy nanoparticles and a corresponding elemental profile acquired across them (red dashed lines). These results together with the one from 50:50 AuAg alloy (cf. Figure 1c in the main text) demonstrate a reliable fabrication of homogenous alloy nanoparticles across the entire range of compositions considered in our work.

\section{Shape Preservation upon Annealing}

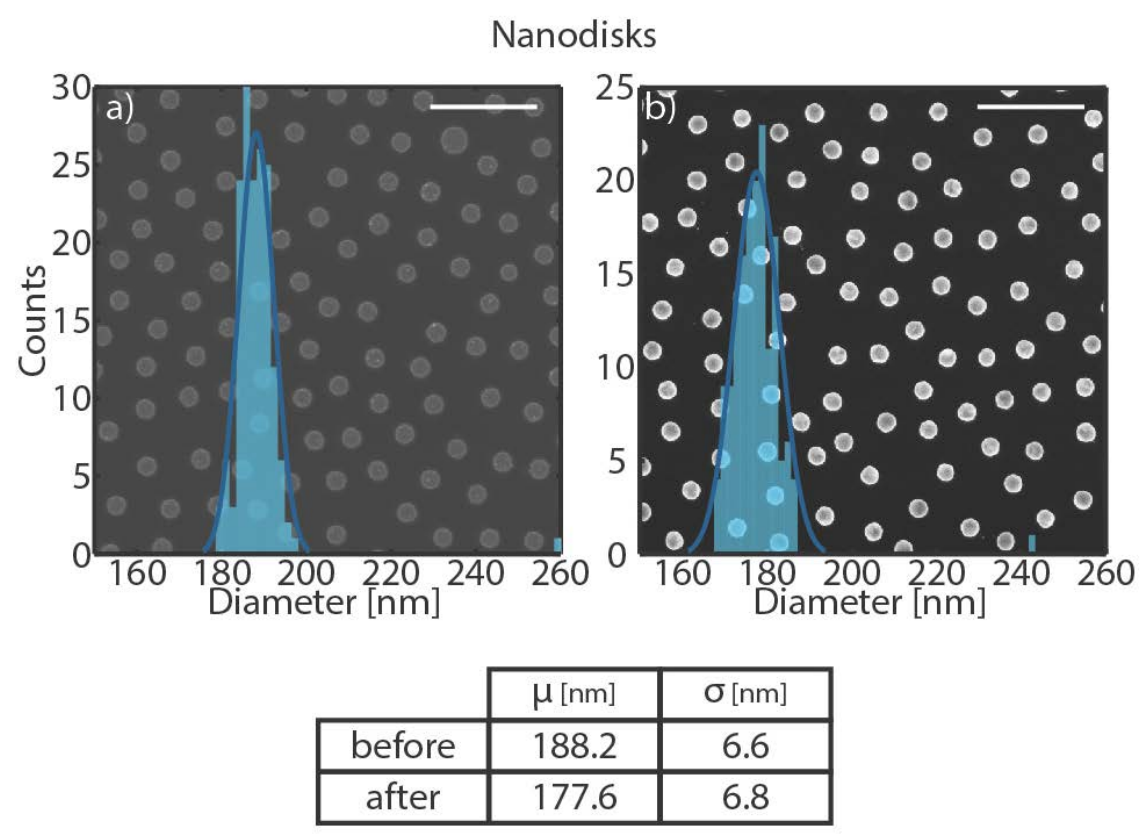

Figure S4. SEM images of AuAg 50:50 nanodisk arrays before (left) and after (right) annealing at $773 \mathrm{~K}$ for $24 \mathrm{~h}$, shown together with their corresponding size distributions. The observed distribution of the unannealed nanodisks is inherited from the polydispersity of the used colloidal particles during fabrication. This size distribution is basically preserved after annealing. Even though the overall diameter slightly shrinks, excellent shape preservation is observed after annealing. Scale bars are $1 \mu \mathrm{m}$. 


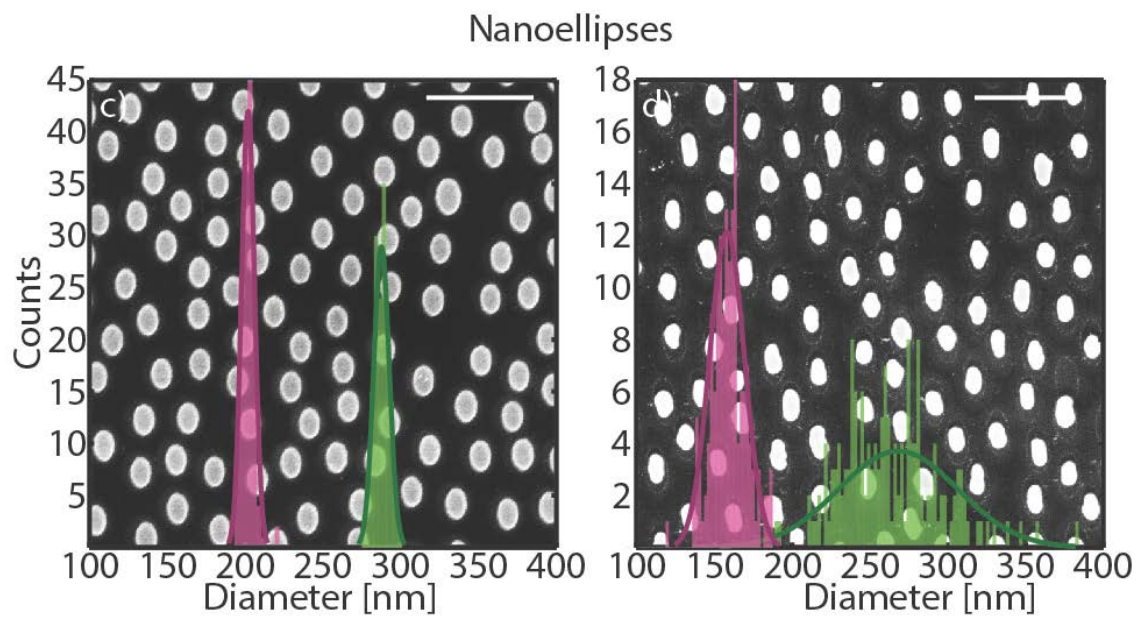

\begin{tabular}{|c|c|c|c|c|c|}
\hline & $\mu$ long [nm] & бlong [nm] & $\mu$ short [nm] & Oshort [nm] & aspect ratio \\
\hline before & 287.7 & 5.0 & 202.0 & 4.2 & 1.4 \\
\hline after & 268.3 & 44.2 & 158.2 & 14.1 & 1.7 \\
\hline
\end{tabular}

Figure S5. SEM images of AuAg 50:50 nanoellipse arrays before (left) and after (right) annealing at $773 \mathrm{~K}$ for $24 \mathrm{~h}$, shown together with their corresponding size distributions. It becomes clear that the anticipated structural anisotropy of the ellipses is retained after annealing. In fact, the anisotropy is even slightly enhanced, as it can be seen by comparing the aspect ratio between ellipse long and short axis. At the same time, a slight change of shape (from more elliptical to more rod-like) is observed, however, without detrimental consequences for the overall purpose of the structure, that is, to exhibit distinct structural anisotropy. Scale bars are $1 \mu \mathrm{m}$.

\section{Hydrogen Sensing}

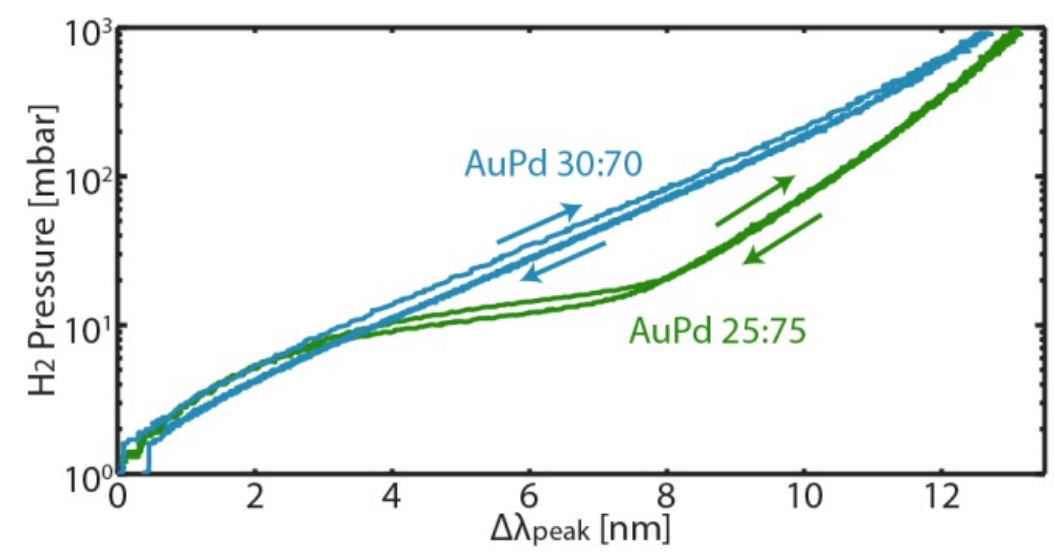

Figure S6. Hydrogen absorption and desorption isotherms of AuPd 30:70 (blue) and 25:75 (green) binary alloy systems. The result is similar to the Figure 7 in the main text but with peak position change $\left(\Delta \lambda_{\text {peak }}\right)$ as readout. The AuPd 25:75 data is adapted from Wadell et al. ${ }^{3}$ 


\section{References}

(1) Fredriksson, H.; Alaverdyan, Y.; Dmitriev, A.; Langhammer, C.; Sutherland, D. S.; Zäch, M.; Kasemo, B. Hole-Mask Colloidal Lithography. Adv. Mater. 2007, 19, 42974302.

(2) Syrenova, S.; Wadell, C.; Langhammer, C. Shrinking-Hole Colloidal Lithography: Self-Aligned Nanofabrication of Complex Plasmonic Nanoantennas. Nano Lett. 2014, 14, 2655-2663.

(3) Wadell, C.; Nugroho, F. A. A.; Lidström, E.; Iandolo, B.; Wagner, J. B.; Langhammer, C. Hysteresis-Free Nanoplasmonic Pd-Au Alloy Hydrogen Sensors. Nano Lett. 2015, 15, 3563-3570. 